\title{
The Integrative Medicine-An Indian Perspective Pivoted on
}

\section{Ayurveda}

\section{Gangadharan GG*}

Ramaiah Indic Specialty Ayurveda - Restoration Hospital, India

Conceptual Paper

Volume 2 Issue 8

Received Date: October 26, 2018

Published Date: November 05, 2018

DOI: $10.23880 /$ jonam- 16000155

Restoration Hospital, Director, Ramaiah Indic Specialty Ayurveda - Restoration Hospital, MSR Nagar, MSRIT Post, Mathikere, Bangalore, Karnataka, India - 560054, Tel: 9448278900; Email: vaidyaggganga@gmail.com

'The intuitive mind is a sacred gift and the rational mind is a faithful servant. We have created a society that honors the servant and has forgotten the gift.'

- Albert Einstein

\section{Abstract}

There is rising global demand for alternatives to mainstream medical system owing to its inability to satisfactorily address all the current healthcare needs. It is making the case for one such alternative model of Integrative Medicine (IM) that has Ayurveda in the pivot and combines other systems like yoga, physiotherapy and certain elements of Allopathy, to be investigated as a feasible model of IM specifically in the context of India. This paper probe into the possibilities and advantages of developing different kind of integrative medicines pivoted on an established traditional healthcare system, taking Ayurveda as an example. This paper postulates the evolution of integrative medicine through a process of summative medicines where entirely two different paradigm of healing system come together to solve a problem.

Keywords: Integrative Medicine; AYUSH; CAM; Yoga; Unani; Siddha; Homeopathy

Abbreviations: NCD: Non-Communicable Diseases; IM: Integrative Medicine; TCM: Traditional Chinese Medicine; TKDC: Traditional Knowledge Digital Library; CAM: Complementary Ayurvedic Medicine.

The great seers of Ayurveda too considered an intuitive mind as an indispensable pre-requisite to appreciate the depths of its theoretical foundations. The Vedas believed in the concept of one global family
(Vasudhaiva Kutumbakam), which pre-supposes the absence of boundaries for knowledge systems like Ayurveda. There is rising global demand for alternatives to mainstream medical system owing to its inability to satisfactorily address all the current healthcare needs. It is making the case for one such alternative model of Integrative Medicine (IM) that has Ayurveda in the pivot and combines other systems like yoga, physiotherapy and 


\section{Journal of Natural \& Ayurvedic Medicine}

certain elements of Allopathy, to be investigated as a feasible model of IM specifically in the context of India.

Currently, the need for such an approach is not widely recognized and consequently no studies (on an impactful scale) on these lines are being contemplated within the sector. It is relevant to health policy makers, open-minded allopathic doctors, votaries of Complementary \& Alternative Medicine (CAM) and notably to the Ayurveda community (0.7 million Ayurveda practitioners) in India. It will be good if we are able to widen the horizon in the interactions with other systems of medicine, especially Allopathy and also nudge a few open minded allopathic doctors and votaries of CAM to think of Ayurveda beyond the boundaries of it being merely an inventory of herbal formulations that can be prospected to be appended to the conventional system of medicine and help them appreciate the depth of its unique philosophies of health and disease.

The $21^{\text {st }}$ century is witnessing a global upheaval of unprecedented proportions in current healthcare models as they struggle to deal with the explosive increase in Non-Communicable Diseases (NCDs), primarily cardiovascular disease, cancer diabetes, chronic respiratory conditions and mental illnesses. Recognizing the magnitude of this problem, the General Assembly of the United Nations met on a health-related issue for the second time in its history in 2011-(the first was in 2001 on HIV/AIDS) to develop a global strategy for the prevention and control of NCDs. The political declaration noted the 'critical importance of strengthening healthcare for people with non-communicable diseases by developing evidence-based norms, standards and guidelines for cost-effective interventions and by reorienting health systems to respond to the need for effective management of diseases of a chronic nature.' The scope of this resolution is sweeping, encompassing all levels of society and requires a fundamental rethinking of concepts of health and disease.

The successes of molecular biomedicine and prevailing drug development strategies have promoted a reductionist search for pathogenic mechanisms at the single molecule/receptor level, which may be inappropriate for NCDs which are being increasingly understood as 'life-style' disorders. Ayurveda, by virtue of its whole-system approach that uses a judicious combination of lifestyle modifications, dietary changes, detox therapies as well as pharmacological disease management for restoration of biological mechanisms is in a unique position to address the challenge of noncommunicable diseases.

Ayurveda is gaining increasing acceptance worldwide, along with other hitherto disregarded systems of health and medicine including Yoga, Homeopathy, Traditional Chinese Medicine (TCM), Acupuncture, etc., (referred to collectively as CAM), evidenced by their inclusion in medical college curricula and establishment of IM centres. Alternative systems of medicine are alive and thriving in India, with Ministry of Ayurveda, Yoga, Unani, Siddha and Homeopathy (AYUSH), Government of India, dedicated to promoting "AYUSH systems as the preferred systems of living and practice for attaining a healthy India."In the Indian context, it makes sense to promote such an integrative model pivoted on Ayurveda as an ideal choice because India has over five millennia of experience with medical knowledge systems like Ayurveda and Yoga. There is a wealth of knowledge including advanced theories of individual health and disease, sophisticated principles of drug design and delivery, a vast repository of knowledge about drugs and their effect on systems, as we are now beginning to understand.

2,00,000 formulations in Traditional Knowledge Digital Library (TKDC) 7,000 Medicinal Plants M\&B GOEF -Ministry of Environment and Forest. Nevertheless, in other parts of the world, other models of IM may evolve into different dimensions of healthcare. Ayurveda may be the pivot in most of India, Nepal and in many parts of South East Asia. However Siddha may be the appropriate pivot for IM in Tamil Nadu and certain pockets in South East Asia, while TCM may play that role in China. Each model may prove relevant and essential to provide healthcare in its respective area. Diabetes Mellitus (DM) is an excellent test case for disc using integrative approaches to the management of non-communicable disorders. Although DM is associated with the least mortality amongst non-communicable diseases, it contributes significantly to disease burden by increasing the risk of cardiovascular disease - the biggest killer of the big four. Global prevalence diabetes in 2011 was estimated at 366 million, projected to increase to 552 million by $2030-32$. Estimated global expenditure due to diabetes was USD 465 billion dollars, amounting to $11 \%$ of total healthcare expenditures in adults (20-79 years). Prevalence of diabetes in India was estimated at 62.4 million, with 77.2 million at extremely high risk of progressing to diabetes. 


\section{Journal of Natural \& Ayurvedic Medicine}

Although discussed primarily as a condition of increased blood sugar (hyperglycaemia), resulting from reduced insulin production or increased body resistance to insulin, diabetes is a multisystem disorder with cardiovascular, neurological, psychiatric, renal and visual complications. Diabetes shares risk factors with all other NCDs-alcohol, tobacco, diet and lifestyle. Furthermore, diabetes can increase the risk of other non-communicable disorders such as cardiovascular disease and stroke, with greater concomitant morbidity and mortality. The conventional strategy of managing a multisystem disorder by referring patients to multiple specialists greatly complicates care and increases costs. Pharmacological management is the first line of treatment, but drugs for complications such as depression may worsen control of blood sugar. Furthermore, reliance on pharmacology may result in ignoring lifestyle modifications, leading to poor treatment outcomes. Alternative treatments are generally not covered by healthcare systems and insurance plans, leading to high out-of-pocket costs.

There is increasing evidence to support a multifactorial approach to diabetes management. Standard regimens of oral anti-glycaemic (sugarlowering) drugs work initially, but have poor long-term efficacy with poor treatment adherence. One response to this issue is to recommend more aggressive pharmacological management, with insulin and drug poly-therapy even in the early stages of the disease. However, a survey of 13 countries in Asia, Europe, Australia and the United States revealed that poor psychological wellbeing may be one of the biggest factors in lack of treatment efficacy, regardless of culture, education or socioeconomic status. Diabetic care providers reported lack of confidence in their ability to diagnose and manage psychological issues.

The importance of diet may have unique implications for India. There is increasing evidence that the etiology and pathogenesis of diabetes may be different in South East Asians, with the description of the "Asian Indian Phenotype", characterized by increased body fat, lower muscle mass and a possible genetic susceptibility to early diabetes (10-15 years earlier than Caucasians) and premature cardiovascular disease. Such a phenotype has increased vulnerability to dietary changes associated with increasing urbanization and industrialization, including excess consumption of calories, increasing meat intake, saturated fats, trans fatty acids, simple sugars, and salt, with low intake of fibre, monounsaturated fatty acids, and n-3 polyunsaturated fatty acids. There has been a progressive reduction in the consumption of pulses, legumes and high-fibre carbohydrates, which tends to increase blood sugar levels. This dietary pattern, coupled with sedentary behaviours promotes obesity, diabetes and cardiovascular disease in both urban and rural areas.

The importance of diet, lifestyle and psychological issues, with the need to consider genetic factors that may be unique to India suggest that any integrative model of medicine should be based on a whole-system approach. Ayurveda may hold great relevance in mitigating the devastating effect of non-communicable disorders in India and globally. Complementary, alternative and IMs are terms being increasingly used in the current scenario of medical pluralism. CAM has been defined largely in relation to conventional biomedicine, depending on whether the practice complements or replaces conventional medicine. The commonly accepted definition states that CAM is a broad domain of healing resources that encompasses all health systems, modalities, and practices and their accompanying theories and beliefs, other than those intrinsic to the politically dominant health system of a particular society or culture in a given historical period. CAM includes all such practices and ideas self-defined by their users as preventing or treating illness or promoting health and wellbeing. Boundaries within CAM and between the CAM domain and that of the dominant system are not always sharp or fixed. The dominant health system of course refers to the conventional Western medical model as it is practiced today.

Ironically, the very same definition may be used for Western medicine in Indonesia, China, Japan and Vietnam, where the so-called 'traditional medicine' is used by the majority of the population. It is also interesting to note that osteopathy, which holds that all diseases can be explained as disorders of the musculoskeletal system and therefore cured by joint manipulation, is considered conventional medicine in the U.S., despite its radical deviance from the principles of biomedicine. In contrast, homeopathy and naturopathy, which also evolved in the West, are given greater professional recognition in the U.K. and in Europe than in the United States.

CAM practices are generally grouped into those administered by practitioners, including herbal medicine, acupuncture, homeopathy, Traditional Chinese Medicine (TCM), chiropractic, naturopathy, osteopathy, Ayurvedic medicine, and massage therapy and self-administered therapy: homeopathy products, herbal supplements, TCM products, naturopathic products, and nutritional products. The diversity of philosophies and 


\section{Journal of Natural \& Ayurvedic Medicine}

methodologies of various systems resist classification and it is highly likely that the very definition of what is conventional, complementary or alternative is susceptible to change.

The 1990s were a landmark decade in the rise of CAM in the west, it was estimated that in the United States visits to CAM providers greatly outnumbered visits to primary care physicians (425 million vs. 388 million), with equivalent out-of-pocket, or non-insured costs ( $\$ 10.3$ billion vs. $\$ 12.8$ billion) in 1990 . Alternative therapies were most commonly used for chronic conditions, including back problems, anxiety, depression, and headaches. The enactment of the Dietary Supplement and Health Education Act (DSHEA) in 1994 to regulate the use of herbal preparations as food supplements and establishment of the office of Alternate Medicine by the National Institutes of Health, which evolved into the National Centre for Complementary and Alternative Medicine (NCCAM), indicate the growing recognition that conventional medicine alone was insufficient to deal with the healthcare needs of the public.

NCCAM's third strategic plan (2011-2015) acknowledges that 'the boundaries between CAM and conventional medicine (also called Western or allopathic medicine) are not absolute.' A WHO survey in 2002 of the prevalence of CAM use revealed up to $80 \%$ use in Africa for primary health needs and was fully integrated into the health system in China (95\% of hospitals), Japan (72\% of physicians), India (2,860 hospitals), Thailand and Vietnam. In the west, CAM was not fully integrated into the healthcare system, but was by $29-42 \%$ in the United States, $49 \%$ in Australia and $75 \%$ had used CAM at least once in France. IM is a new concept where the modern biomedical system is trying to integrate aspects of traditional systems in areas of its weakness. In most of these models the integration was only appropriation or misappropriation of certain elements of traditional medicines without going into its philosophy. Indeed it has been argued that IM is "... not the changing of conventional medicine but the taming of CAM". One example of truly IM is the work of Dr. Dean Ornish to apply the practice of yoga and dietary changes according to Ayurveda to reversal of conventionally diagnosed cardiovascular disease without drugs or surgery, which had a profound impact on conventional medicine. There has been a shift in medical practice towards treatments that are evidence-based regardless of medical tradition, despite increasing resentment and campaigns against integrating CAM with conventional medicine. There is therefore a growing need to revitalize and promote
Ayurveda and such other Traditional Medicine (TM) systems for their contemporary relevance with the spirit of a dispassionate scientific enquiry. It is however important to recognize that Ayurveda is not merely meant to be used as an inventory for prospecting new molecules or extracts, but it is also to be explored for its sophisticated theory of homeostasis and bio-regulation.

Historically, Ayurveda has been a holistic, inclusive, progressive and continuously evolving knowledge system with universal attributes. The logic, theoretical foundations and epistemology of Ayurveda are based on the six darśanas, mainly the Sānkhya and Nyaya-Vaisesika systems of natural philosophy. An integrative holistic approach to healthcare and cure has been a distinct facet of Ayurveda practice. On the other hand, critical care and advanced invasive treatments are great contributions of modern medicine to healthcare, while for curative care, its approach has been reductionist, which many biomedical scientists believe, does not probe the root of the disease. A careful scrutiny of the classical writings of Ayurveda will enable us to appreciate the rational and scientific development of medical knowledge in India. It will help us know the reason why genuine practitioners of Ayurveda who have relied entirely on classical medicines invariably claim to have got clinical outcomes that were predicted of them, ages ago.

Nevertheless, one cannot undermine the need for clinical evidence in a form discernible to the conventional scientific community when it is evident that no single system can claim to offer a complete cure. In this age of globalization of information where people have access to the entire body of medical literature at the click of a button, they can be expected to judge for themselves the good in every system and resort to them as they deem suitable. Just as critical care and invasive procedures of contemporary medicine are evidently helpful, Ayurveda can make two major contributions in the current healthcare scenario. One is in the management of chronic ailments (like Diabetes, Bronchitis, Stress etc.), and second one in home based remedy for primary healthcare.

\section{Conclusion}

It has been a long recognized need that India should take up the initiative of developing a healthcare model pivoted on Ayurveda by integrating traditional and modern systems. As a first step it should attempt to look for commonalities in the various traditional practices like Ayurveda, Siddha, folk medicines etc. and then invest in rigorous clinical documentation of their clinical outcomes 


\section{Journal of Natural \& Ayurvedic Medicine}

in an integrative framework that is made available in a form that can be accessed and understood by any modern scientist. While medical pluralism may be the future direction and indeed the new form of healthcare, the shift from singular knowledge systems to new plural systems cannot happen without very serious and long term investment in research, followed by comprehensive educational programs. India can be a world leader in this new field of 'Integrative Health Sciences and Technology' because we have over the last century or so assimilated and achieved a reasonable degree of competence in biomedical and life sciences and we possess an incredibly rich medical heritage of our own. Revitalization of the TM sector is essential for this leadership role.

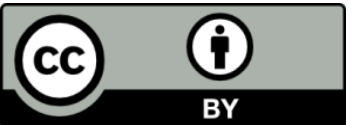

